\title{
Erratum to: Efficiency based strategy spreading in the prisoner's dilemma game
}

\author{
S. Weber ${ }^{\mathrm{a}}$ and M. Porto ${ }^{\mathrm{b}}$ \\ Institut für Festkörperphysik, Technische Universität Darmstadt, Hochschulstr. 8, 64289 Darmstadt, Germany
}

Eur. Phys. J. B 69, 599 (2009)

Received 22 July 2009

Published online 17 September 2009 - (C) EDP Sciences, Società Italiana di Fisica, Springer-Verlag 2009

In the course of extending our recent work [1] to more general game matrices we identified an inequality different from equation (4) of reference [1] for the payoff per interaction case. If the parametrization of the prisoner's dilemma is chosen, the correct threshold condition for the cooperation strategy to be an $\mathrm{ESS}_{N}$ on a vertex with degree $k$ is instead

$$
b<\frac{2 k-4}{2 k-1}
$$

As the temptation to defect parameter $b$ is always greater than 1 , this condition is never fulfilled in the prisoner's dilemma game. Nevertheless, the main argument given in reference [1], that the vertices with the largest degree in the network loose their ability to support cooperation at a certain temptation to defect parameter value $b_{\mathrm{c}}$, is however correct and supported by the numerical data. This can be seen by considering the second moment of degrees of cooperators $\kappa^{2}$ in the stationary state as shown in Figure 1. Our analysis reveals that the second moment $\kappa^{2}$, which is dominated by vertices with a large degree, declines rapidly as $b$ gets larger than $b_{\mathrm{c}}$. This indeed shows that the largest degree vertices cease to be cooperators for $b>b_{\mathrm{c}}$, and successively vertices with smaller degree do so as well as $b$ further increases.

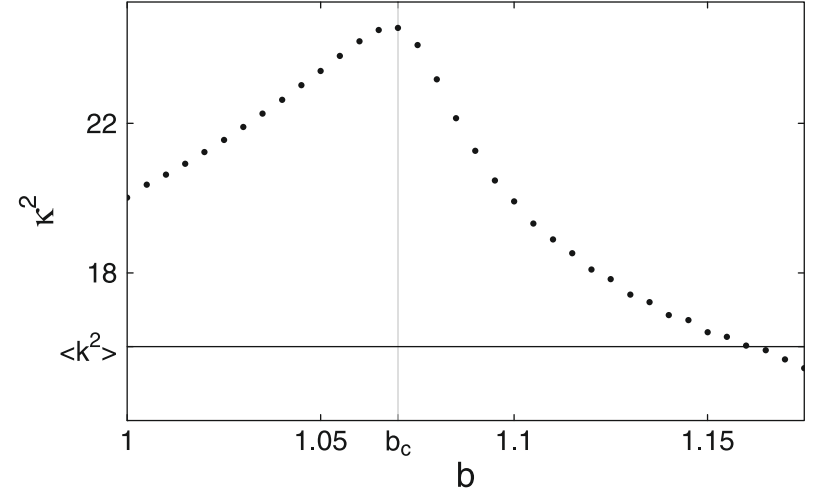

Fig. 1. Second moment of degrees of cooperating players $\kappa^{2}$ for the payoff per interaction case. Shown are the results for scalefree networks with scaling exponent $\gamma=3$ and 4000 vertices. The horizontal line indicates the second moment $\left\langle k^{2}\right\rangle$ of the whole network.

We acknowledge helpful discussions with Christian Kexel.

\section{References}

1. S. Weber, M. Porto, Eur. Phys. J. B 69, 599 (2009)

\footnotetext{
a Present address: Freiburg Institute for Advanced Studies (FRIAS), University of Freiburg, 79104 Freiburg, Germany.

b e-mail: porto@fkp.tu-darmstadt.de
} 